\title{
Performance Enhancement and Comparison of Graphene Field Effect Transistor Devices Coated with HMDS Layer
}

\author{
Manoharan Arun Kumar ${ }^{1,2}$ (D) $\cdot$ Ramasamy Jayavel $^{2} \cdot$ Mukannan Arivanandhan $^{2} \cdot$ Balwinder Raj $^{3} \cdot$ N. Mohankumar ${ }^{1}$
}

Received: 13 December 2021 / Accepted: 15 February 2022 / Published online: 7 March 2022

(C) The Author(s), under exclusive licence to Springer Nature B.V. 2022

\begin{abstract}
Graphene-based devices show good transfer characteristics, which depend upon the surface morphology of the material and substrate. During fabrication of the device, the substrate morphology is disturbed inappropriately by the surface contamination. In the present study, monolayer Graphene Field Effect Transistor (GFET) has been driven with hydrophobic Hexamethyl Disilazane (HMDS) layer. The HMDS layer is dehydrated before and after the exfoliated monolayer graphene, and the electrical characteristics were measured. The transfer curve of the HMDS coated graphene device demonstrates excellent FET characteristics and prevents contamination from the atmosphere under ambient conditions. Fabrication of GFET device on the hydrophobic substrate enhances the effective ambipolar behavior. It is beginning with an excellent platform and user-friendly device for biosensing applications.
\end{abstract}

Keywords Graphene $\cdot$ Hexamethyl Disilazane (HMDS) $\cdot$ Atomic layer deposition (ALD) $\cdot$ Dirac point $\cdot$ Ambipolar behavior

\section{Introduction}

Graphene $[1-7]$ is a rapidly growing two-dimensional one atom thick carbon material with great potential in linear dispersion [1, 2]. Presently, graphene-based electronic devices [3] are known for attractive peculiarities, like the quantum hall effect [4], tunneling effect [5], and many other transport properties as well as electronic properties [6,7], mechanical properties [8], and optical properties $[9,10]$ owing to their carrier density and polarity, as well as high charge mobility for both electrons and holes. The electronic properties of graphene make it one of the promising materials in generating high-speed sensor devices [11-13]. Graphene is a single atomic layer thick though visible to the naked eye due to its high opacity. Due to Pauli blocking, the optical energy of graphene is comparatively less than Fermi

Manoharan Arun Kumar manokavi2011@gmail.com

1 Department of Electrical, Electronics and Communication Engineering, School of Technology, Gandhi Institute of Technology and Management (GITAM), Bengaluru 561203, India

2 Centre for Nanoscience and Technology, Anna University, Chennai, Tamil Nadu 600 025, India

3 Department of Electronics and Communication Engineering, NITTTR, Chandigarh 160019, India energy; hence it becomes transparent. Therefore, graphene would be appropriate for various controllable photonic devices [14]. Another extraordinary property of graphene has been exploited on its mechanical strength. The carbon-carbon bond length of about $0.142 \mathrm{~nm}$ makes graphene the strongest material of about $130 \mathrm{GPa}$, comparatively stronger than A36 steel [15]. Graphene has been used in different platforms such as fieldeffect transistor [16, 17], supercapacitors [18, 19], solar cells [20, 21], biosensors [22-27] and nanoelectronic devices [28]. The graphene field-effect transistor was fabricated on $\mathrm{SiO}_{2}$ substrate by the Chemical Vapor Deposition (CVD) [12, 20, 22] and mechanical exfoliation method [28]. Among these methods, the exfoliated graphene films onto the thermally oxidized silicon substrates show the best quality and most attractive for electronic devices to date. Undesirably, the transfer characteristics of those devices may vary widely and exhibit a large variance in the mobility and the ambipolar behavior between the electron and hole transport of the devices [29]. The developed technology still lacks to overcome the difficulties like contamination and substrate deficiencies during the fabrication process. Especially, the imperfection and poor reproducibility of the devices have occurred due to the molecules present in the ambient air [30]. In such cases, the self-annealing process [3] reduces the substrate effects. The substrate was annealed at sufficient temperature during the annealing process, making the substrate-free from contaminants. To identify a suitable approach, three different 
possible methods have been explored and compared to obtain good ambipolar behavior in the transfer characteristics of the devices. In the first method, annealing process was used to fabricate the device, with substrate annealing at a certain temperature. Herein, the samples were prepared in the absence of hydrophobic Hexamethyl Disilazane (HMDS, Merck Performance Materials, Darmstadt, Germany) layer [11, 31] and Atomic Layer Deposition (ALD) of $\mathrm{Al}_{2} \mathrm{O}_{3}[11,32]$. In the second method, the samples were prepared with the deposition of the HMDS layer before graphene exfoliation. In the third method, the samples were prepared with the deposition of the HMDS layer prior and after the graphene exfoliation. From the experimental observations, it was observed that the HMDS layer and $\mathrm{Al}_{2} \mathrm{O}_{3}$ deposition effectively control the contamination of the substrate from ambient air and improve the transfer characteristics and reproducibility of the devices. The advantage of this method is its improved device characteristics.

\section{Materials and Methods}

\subsection{Graphene Deposited on the $\mathrm{SiO}_{2}$ Substrate}

Graphene was exfoliated mechanically from the natural graphite (Covalent Material Co., Ltd., Tokyo, Japan) using adhesive tape and deposited onto the $\mathrm{SiO}_{2} / \mathrm{Si}$ Substrate (90 nm). Optical microscopy (S8 APO + EC3, Leica Microsystems, Wetzlar, Germany) was used to identify the monolayer graphene on the substrate and was confirmed by Raman spectroscopy (RAMANplus, Nanophoton, Osaka, Japan) for all the three possible methods of fabrication. The contact pads were made for the corresponding monolayer graphene, and it has been designed using CAD software (Vectorworks 2012 tool, A\&A Co. Ltd., Tokyo, Japan).

\subsection{Fabrication of GFET Using Annealing Method}

The LOR5A (Microchem Corp., MA, USA) solution was deposited on the substrate as a protective layer and baked at $180{ }^{\circ} \mathrm{C}$ for 5 mins. Then, the AZ5214E (Merck Performance Materials) solution, which acts as the photo resistant coating, was deposited on the substrate and baked at $110^{\circ} \mathrm{C}$ for $2 \mathrm{mins}$. The maskless scanning lithography carried out the etching process (DL-1000/NC2P, NanoSystem Solutions Inc., Okinawa, Japan) with a power of $1 \mathrm{~W} / \mathrm{cm}^{2}$ at $405 \mathrm{~nm}$ and an exposure dosage of $85 \mathrm{~mJ} / \mathrm{cm}^{2}$ to pattern the A-Z resist. The source and drain electrodes (Ti/Au 10/200 nm) were formed by E-gun evaporation (RDEB-1206 K, RDEC Inc. Ibaraki, Japan) and lift-off techniques. The detailed fabrication process was explained in the previous work [11]. To obtain a suitable approach for developing the device, with good ambipolar behavior, three possible methods were followed.
Up to the lift-off techniques, steps followed to make the source and the drain was standard for all three forms.

In the first method, the annealing process was carried out after the lift-off process, and the schematic diagram of the fabrication process is shown in Fig. 1a. During annealing, the substrate was kept for rapid thermal annealing $(\mathrm{QHC}$ $\mathrm{P} 410$ ) at $300{ }^{\circ} \mathrm{C}$ to prevent the substrate from impurities and to reduce the contaminants. Herein, the rising temperature rate was set at less than $40^{\circ} \mathrm{C} / \mathrm{s}$, and the rate of falling temperature was set at $10^{\circ} \mathrm{C} / \mathrm{s}$. The annealing process was carried out in the atmosphere of $\mathrm{N}_{2}$ and $3 \% \mathrm{H}_{2}+\mathrm{Ar}$. The $3 \% \mathrm{H}_{2}+\mathrm{Ar}$ atmosphere was used for cleaning, while the $\mathrm{N}_{2}$ atmosphere was helpful in cooling. Figure $1 \mathrm{~b}$ demonstrates the monolayer graphene on bare $\mathrm{SiO}_{2}$, and Fig. 1c shows the fabrication of electrodes on the bare substrate during the annealing process. Therefore, the samples were prepared in the atmosphere mentioned above, which can control substrate contamination without the HMDS and ALD- A12O3 layers.

\subsection{Fabrication of GFET Using HMDS Layer and ALD Deposition}

In the second method, the GFET device was fabricated by the graphene deposited on the HMDS layer, and the schematic diagram of the fabrication process is shown in Fig. 2a. The HMDS layer is self-assembled. Herein, the HMDS layer was coated before the exfoliated graphene. Then, the substrate was prepared in the same fashion up to the lift-off process as shown in Fig. $2 \mathrm{~b}$ and c. The ALD- $\mathrm{Al}_{2} \mathrm{O}_{3}$ insulating layer was deposited on electrodes at $0.8 \AA /$ cycle, and it is shown in Fig. $2 \mathrm{~d}$.

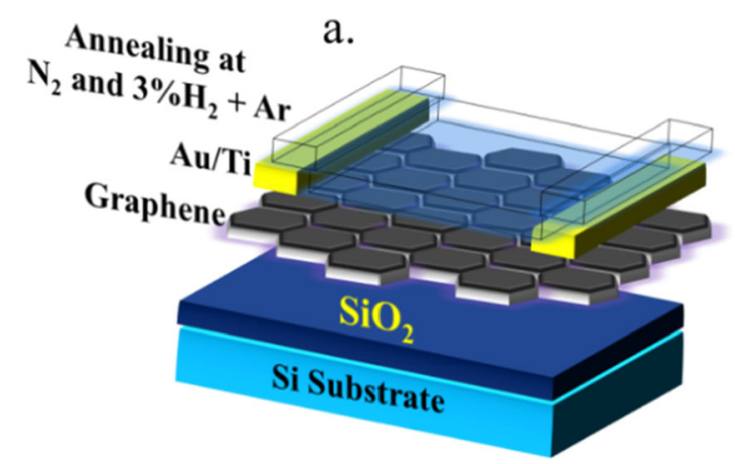

b.



Fig. 1 a. A Schematic diagram of the fabrication process by annealing method, b. Optical microscopic image of exfoliated graphene on $\mathrm{SiO}_{2}$ layer, c. Fabrication of source-drain electrodes $(\mathrm{Ti} / \mathrm{Au})$ 
Fig. 2 a. A Schematic diagram of the fabrication process by HMDS layer and ALD deposition, $\mathbf{b}$. Optical microscopic image of exfoliated graphene on $\mathrm{SiO}_{2}$ layer, c. Fabrication of sourcedrain electrodes $(\mathrm{Ti} / \mathrm{Au}), \mathbf{d}$. After ALD- $\mathrm{Al}_{2} \mathrm{O}_{3}$ deposition b.

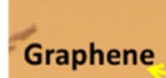

$\mathrm{SiO}_{2}(90 \mathrm{~nm})$

\section{a.}

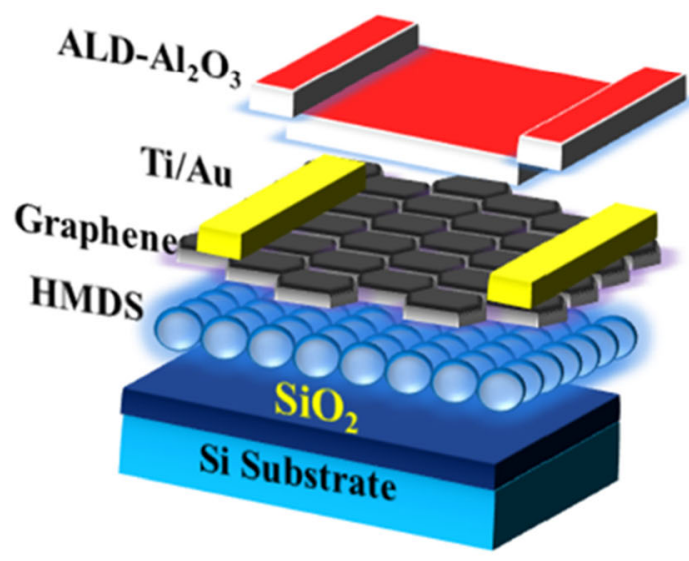

c.

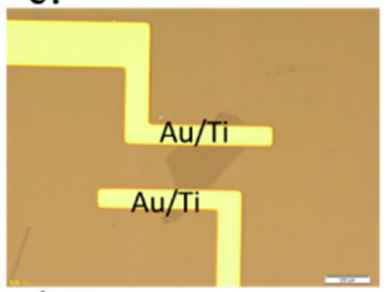

d.



\subsection{Fabrication of GFET Using Prior and after Deposition of HMDS on Graphene Exfoliation and ALD Deposition}

In the third method, the substrate was coated with HMDS before the exfoliated graphene and deposition of the insulating

Fig. 3 a. A Schematic diagram of the fabrication process by prior and after deposition of HMDS and ALD deposition, b. Optical microscopic images of exfoliated graphene on $\mathrm{SiO}_{2}$ layer, $\mathbf{c}$.

Fabrication of source-drain electrodes (Ti/Au), d. After ALD$\mathrm{Al}_{2} \mathrm{O}_{3}$ deposition

a.

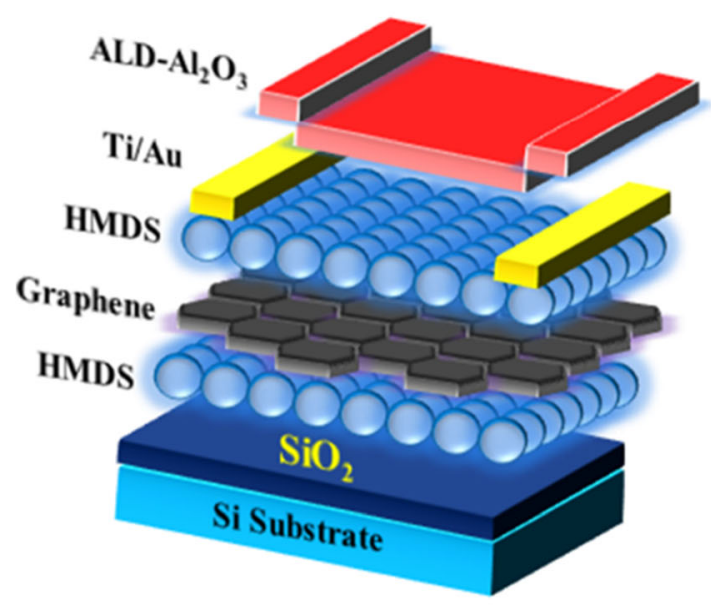

b.

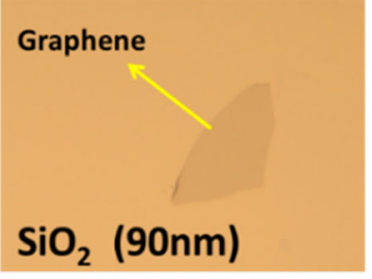

c.

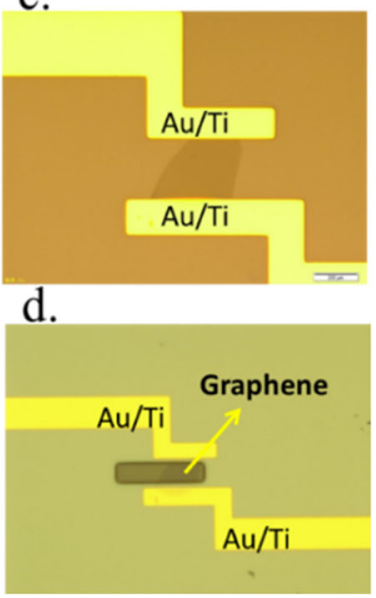




\section{Results and Discussion}

\subsection{Transfer Characteristics of GFET Using Annealing Method}

In this study, the GFET device was fabricated without $\mathrm{Al}_{2} \mathrm{O}_{3}$ and HMDS layers. The formation of single-layer graphene was identified by means of optical microscopy and Raman spectroscopy. The source and drain electrodes were fabricated using the maskless scanning lithography, E-gun evaporator, and lift-off method. The output and transfer characteristics of the device were measured by using the kiethley parametric analyzer. As shown in Fig. 4a, the output characteristics (Id Vs $V_{\text {bias }}$ ) were measured by sweeping voltage in the range from $-30 \mathrm{~V}$ to $+30 \mathrm{~V}$ with a step voltage of $5 \mathrm{~V}$. The output characteristics show the linear behavior at zero bias which illustrates good metal contacts with graphene.

The contact pads have been designed on the substrate by using CAD software. The transfer characteristics of the device were measured at $\mathrm{V}_{\mathrm{d}}=0.01 \mathrm{~V}$, and $\mathrm{V}_{\mathrm{gs}}$ was set -30 to $+30 \mathrm{~V}$ as shown in Fig. 4b. The I-V characteristics of graphene show a gradual decrease during the hole transport region and then increase during the electrons transport region. Pioneering transport characteristics have already been reported on graphene sheets. For instance, Ohno et al. [33] proposed the transistor characteristics with the different fabrication processes. In Fig. 4b, the graph shows the Id of the bare graphene, which has a gradual decrease in hole transport but increases to a certain level in electron transport. The obtained result shows the lower ambipolar behavior. The gradual decrease and increase represent ambipolar behavior due to holes in the negative voltage and electrons in the positive voltage. Here, the conduction of electrons on positive voltage is poor. It has been suggested that the changed impurities may cause poor ambipolar behavior. Due to the poor ambipolar behavior, the sharpness of the peak at the Dirac point becomes broader. Both holes concentration and electron concentration were neutralized at Dirac point. The corresponding resistance curve of the graphene device measured at $\mathrm{V}_{\mathrm{d}}=0.01 \mathrm{~V}$ as shown in Fig. 4c.

\subsection{Transfer Characteristics of GFET Using HMDS Layer and ALD Deposition}

GFET devices with exfoliated graphene on $\mathrm{SiO}_{2}$ exhibit high quality. Unfortunately, the characteristics widely change due to the changes in ambient conditions and asymmetries between electrons and holes transportation.

However, the technologies cannot control the contamination during the processing and absorption of ambient air molecules. The samples were prepared under ambient conditions, imbibing the atmosphere's water molecules. It has been revealed previously in the annealing method the substrates are kept in $300{ }^{\circ} \mathrm{C}$ to remove the contaminants such as $\mathrm{H}_{2} \mathrm{O}$ and $\mathrm{CO}_{2}$, which also reduces the doping level. Though the samples a.



b.

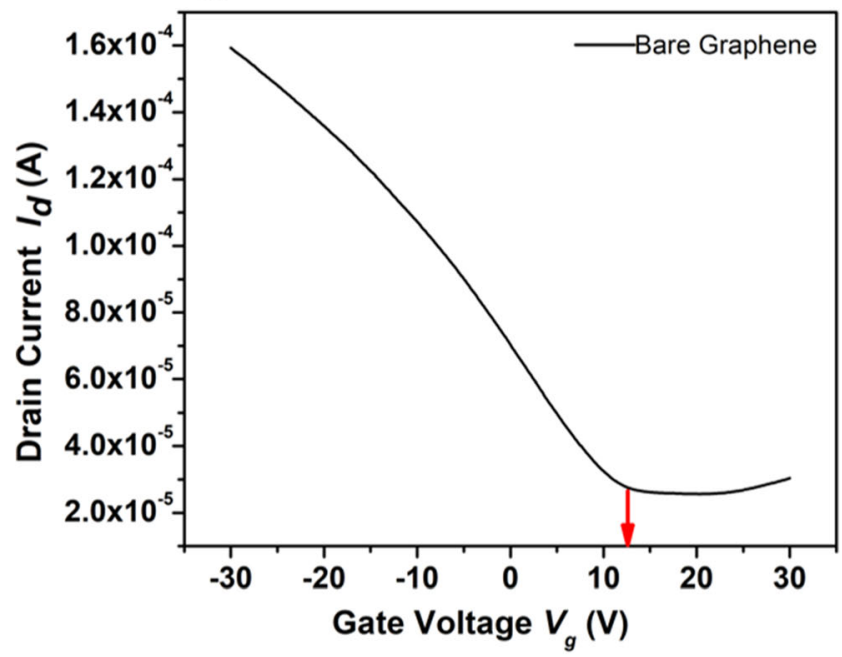

C.

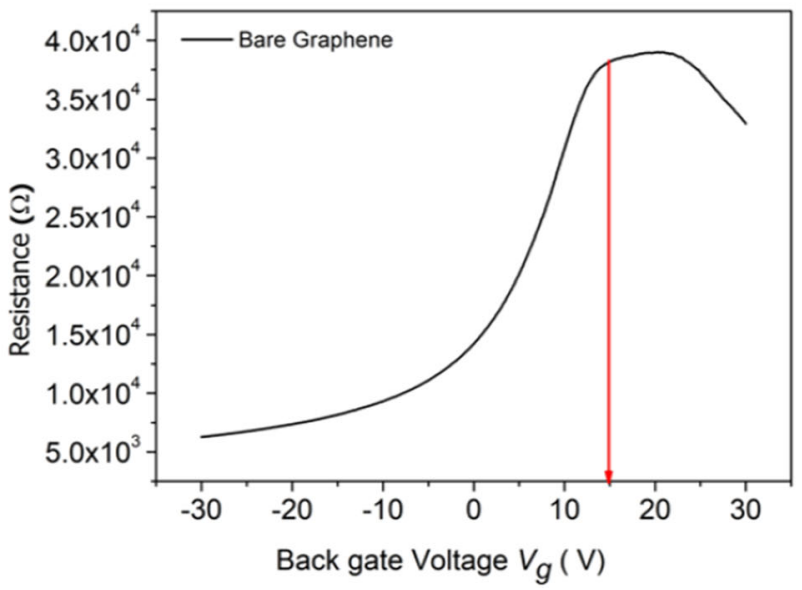

Fig. 4 The I-V characteristics of the back gated graphene device using annealing process, a. Output characteristics with drain current vs. bias voltage measured at various $\mathrm{V}_{\text {gate }}$ ranging from $-10 \mathrm{mV}$ to $10 \mathrm{mV}$ with step of $0.01 \mathrm{~V}, \mathbf{b}$. The transfer curve for bare graphene between drain current vs. back gate voltage at $\mathrm{V}_{\mathrm{d}}=0.01 \mathrm{~V}$, c. The corresponding resistance curve of the graphene device measured at $\mathrm{V}_{\mathrm{d}}=0.01$ 
a.

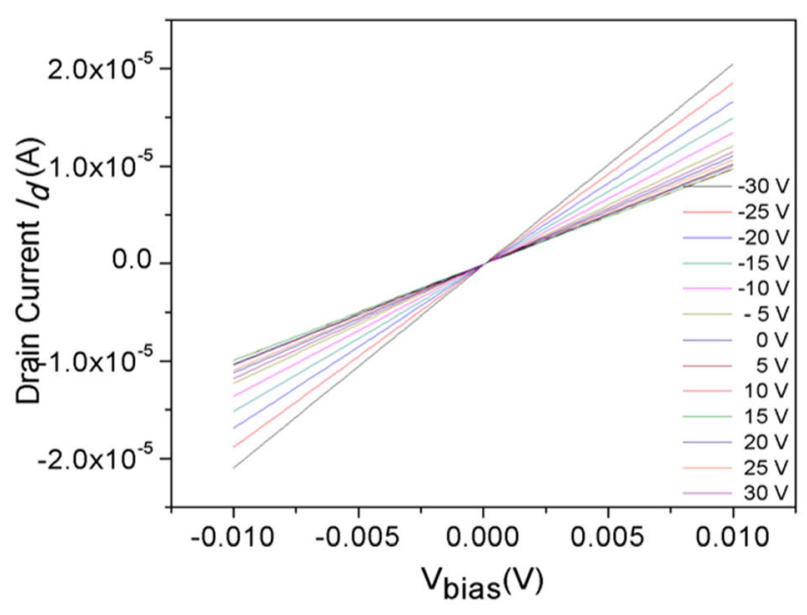

b.



c.

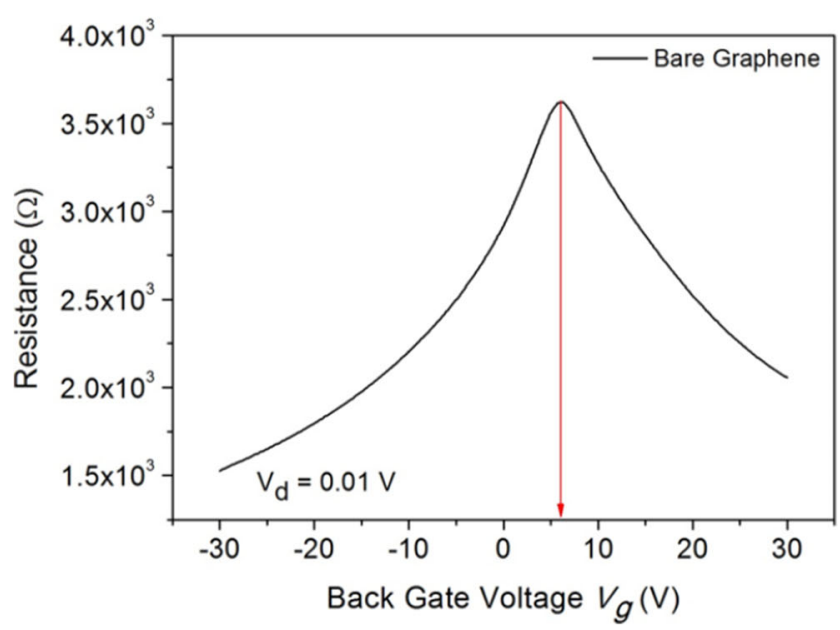

were prepared so that when exposed to air, the samples recuperate the same doping level, as argued by Lafkioti et al. [30], the contaminants were still observed on the graphene surface.
Fig. 5 The I-V characteristics of back gated graphene device using HMDS layer and ALD deposition, a. Output characteristics with drain current vs. bias voltage measured at various $\mathrm{V}_{\text {gate }}$ ranging from $-10 \mathrm{mV}$ to $10 \mathrm{mV}$ with step of $0.01 \mathrm{~V}$, b. The transfer curve for bare graphene between drain current vs. back gate voltage at $\mathrm{Vd}=0.01 \mathrm{~V}$, c. The corresponding resistance curve of the graphene device measured at $\mathrm{Vd}$ $=0.01 \mathrm{~V}$

Moser et al. [34] also reported that the samples prepared similarly during the annealing method might have defects such as edges, wrinkles, etc., which were present as a static site on the surface of graphene along with the contaminants. These imperfections and poor reproducibility play a crucial role in fabricating the GFET devices.

From the experimental observation of the device (Fig. 4), the fabrication by annealing was not much practical for the surface quality. The $\mathrm{SiO}_{2}$ substrate surface quality is more important to impose the exfoliated graphene. The surface quality can be improved by a suitable approach named chemical cleanliness. The $\mathrm{SiO}_{2}$ layer was carefully cleaned in N-Methyl-pyrrolidone, acetone, and 2-proponal. Before exfoliation of graphene on the $\mathrm{SiO}_{2}$ layer, the substrate was exposed to HMDS solution for $24 \mathrm{~h}$. The molecules present in the HMDS solution play a vital role in the self-assembled graphene on $\mathrm{SiO}_{2}$. The exfoliated graphene flakes on HMDS were observed using Raman spectra and Optical microscopy. The color and morphology do not differ much from graphene on HMDS and bare graphene without HMDS.

The output characteristics of the device are shown in Fig. 5a. Significant changes can be observed in ambipolar behavior from the devices' transfer characteristics, as shown in Fig. 5b. The results show proper conduction of holes and electrons with a gradual decrease in holes transport and an increase in electron transport. The sharpness of the peak at the Dirac point has also improved with proper charge carrier conversion. It has been suggested that the charge impurities were reduced by the HMDS layer as well as charged improvement in the FET characteristics [31]. The corresponding resistance curve of the graphene device measured at $\mathrm{V}_{\mathrm{d}}=0.01 \mathrm{~V}$, as shown in Fig. 5c. The $\mathrm{Al}_{2} \mathrm{O}_{3}$ layer also plays a vital role in avoiding further contamination from ambient air molecules during measurements. It increases the surface passivation of the substrate. Primarily, it is helpful in the gate electrode used for sensing applications.

For the sake of comparison, the reference samples were prepared without HMDS layer and with HMDS layer deposited prior to the exfoliation graphene. From the comparison results, the graphene deposition with HDMS layer samples shows good transfer characteristics, and it is more beneficial for sensing applications such as DNA hybridization [11], $\mathrm{pH}$ sensor [27, 35], etc. 
a.

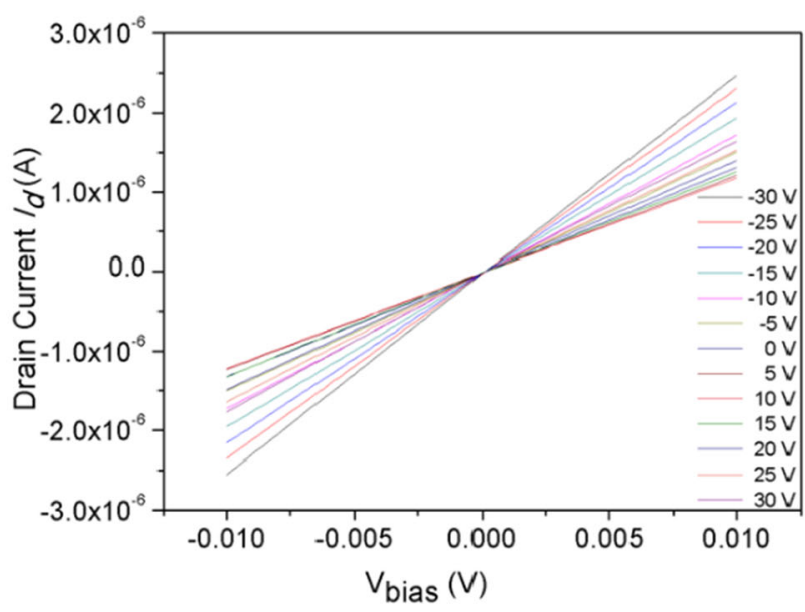

b.

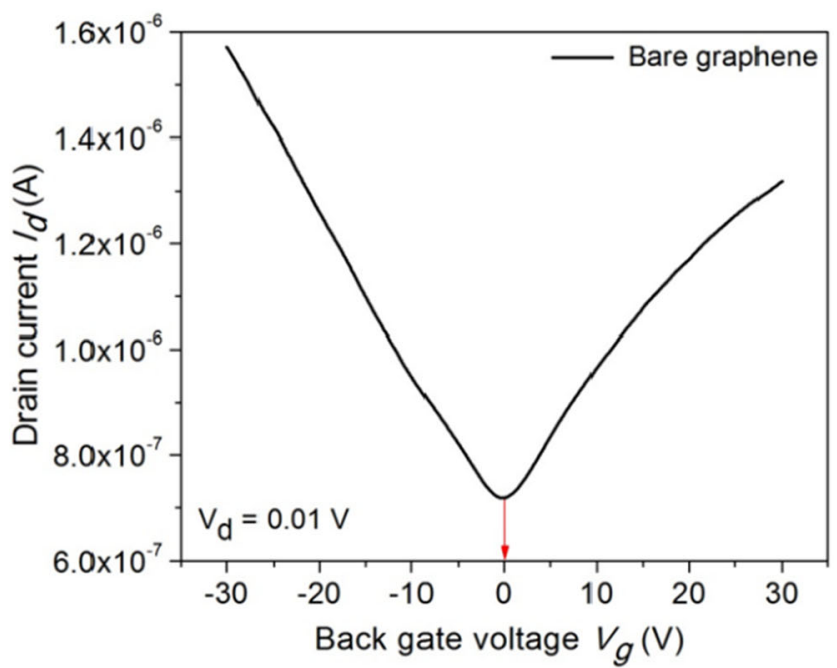

c.

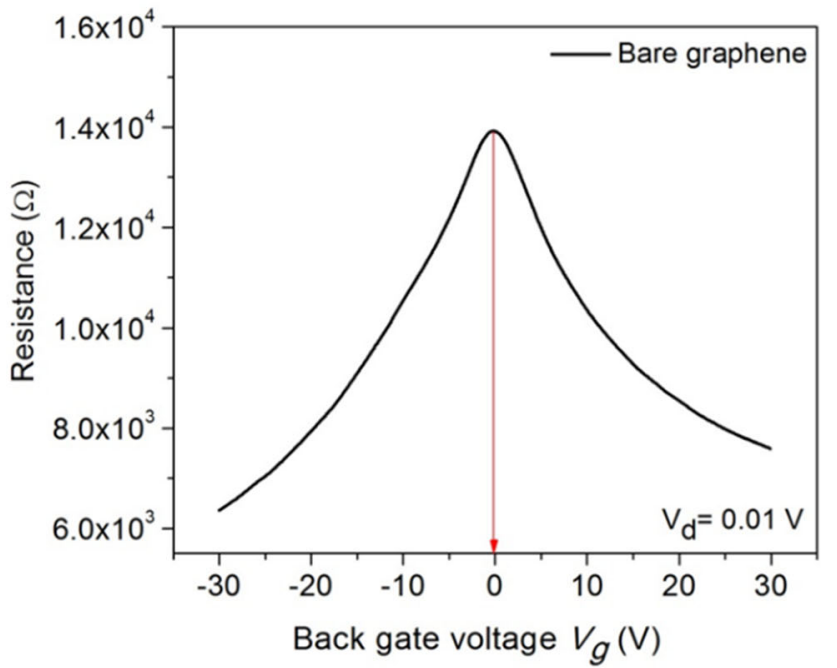

Fig. 6 The I-V characteristics of the back gated graphene device using prior and after deposition of HMDS and ALD deposition. a. Output characteristics with drain current vs. bias voltage measured at various $\mathrm{V}_{\text {gate }}$ ranging from $-10 \mathrm{mV}$ to $10 \mathrm{mV}$ with step of $0.01 \mathrm{~V}$. b. The transfer curve for bare graphene between drain current vs. back gate voltage at $\mathrm{V}_{\mathrm{d}}=0.01 \mathrm{~V}$. c. The corresponding resistance curve of the graphene device measured at $\mathrm{V}_{\mathrm{d}}=0.01 \mathrm{~V}$

\subsection{Transfer Characteristics of GFET Using Prior and after Deposition of HMDS on Graphene Exfoliation and ALD Deposition}

The self-assembled layer of HMDS before graphene exfoliation has reduced the impurities and improved the ambipolar behavior and electron conduction. Further, to improve the quality of graphene and its characteristics, a suitable approach has been identified. In addition to the self-assembled layer of HMDS molecules before graphene deposition on the substrate, the HMDS molecules were deposited on the exfoliated graphene flakes. Then, the fabrication process was started with LOR5A (protective layer) and AZ5214E (photoresist layer). Subsequently, the substrate was treated with an HMDS layer (which acts as a bed) for graphene with a strong hydroxyl group (-OH). It forms a hydrophilic dangling bond for both silicon and graphene as a silanol layer. It improves the interaction between the molecules and the substrate, making the hole and electron conduction in exfoliated graphene effective. The output characteristics of the device are shown in Fig. 6a. The transfer characteristics of the device with a sharp resistance peak are obtained at the Dirac point, as shown in Fig. 6b. From Fig. 6, it is observed that the Dirac point achieved for the gate voltage at $\mathrm{Vg}=0 \mathrm{~V}$ indicates that the chemical doping present in the grouping was minimum. Hence, graphene prepared on the HMDS layer with $\mathrm{Al}_{2} \mathrm{O}_{3}$ deposition showed high purity having good transfer characteristics. The corresponding resistance curve of the graphene device measured at $\mathrm{V}_{\mathrm{d}}=0.01 \mathrm{~V}$, as shown in Fig. $6 \mathrm{c}$.

\section{Conclusion}

The graphene fabricated on $\mathrm{Si} / \mathrm{SiO}_{2}$ substrate treated with the thin layer of $\mathrm{HMDS}$ and $\mathrm{ALD}-\mathrm{Al}_{2} \mathrm{O}_{3}$ deposition provides venerable transport characteristics. The main facts are the production of substrates with the HMDS layer having an excellent graphic surface without edges and wrinkles. During ambient conditions, the water molecules in the atmosphere and contaminants such as $\mathrm{H} 2 \mathrm{O}$ and $\mathrm{CO} 2$ cannot attach to the hydrophobic hexamethyl disilazane. Hence, the graphene surface of the device becomes free from pollutants. Therefore, the 
hydrophobic layer makes the graphene-based devices with higher transport properties and improved FET characteristics and good mobility even under ambient conditions. These improved FET characteristics can be used for biosensor applications such as DNA hybridization detection, $\mathrm{pH}$ sensor, protein detection, glucose sensor, etc. The platform proposes a promising way to fabricate high-performance graphene devices for sensor applications.

Acknowledgements One of the authors (M.A.) would like to thank the National Institute for Materials Science (NIMS), Japan, and Anna University, India, for the award of the International Co-operative Graduate School (ICGS) Fellowship. This study was supported by NIMS Molecule and Material Synthesis Platform in the "Nanotechnology Platform Project" operated by the Ministry of Education, Culture, Sports, Science and Technology (MEXT), Japan.

Author Contribution Manoharan Arun Kumar, Ramasamy Jayavel and Mukannan Arivanandhan: Conceptualization; Manoharan Arun Kumar, Ramasamy Jayavel and Mukannan Arivanandhan: investigation; Manoharan Arun Kumar, Ramasamy Jayavel and Mukannan Arivanandhan, Balwinder Raj, N.Mohankumar: resources; Manoharan Arun Kumar, Ramasamy Jayavel: Original draft preparation; Manoharan Arun Kumar, Ramasamy Jayavel and Mukannan Arivanandhan: review and editing; Manoharan Arun Kumar, Ramasamy Jayavel and Mukannan Arivanandhan, Balwinder Raj, N.Mohankumar: Visualization; Ramasamy Jayavel: Supervision;

Data Availability No supplementary materials.

\section{Declarations}

Authors Agreement We declare that the manuscript entitled "Performance Enhancement and Comparison of Graphene Field Effect Transistor devices coated with HMDS layer" is original, has not been fully or partly published before, and is not currently being considered for publication elsewhere. We confirm that all named authors have read and approved the manuscript. We further ensure that the undersigned has approved the order of authors listed in the manuscript. We understand that the Corresponding Author is the sole contact for the editorial process. The corresponding author "Dr. Manoharan Arun Kumar " is responsible for communicating with the other authors about the process, submissions of revisions, and final approval of proofs."

Human and Animal Rights This article does not contain any studies with human or animal subjects.

Ethics Approval and Consent to Participate Authors freely agreed and gave their consent for the publication of the paper.

Conflict of Interest The authors declare that they have no conflict of interest.

\section{References}

1. Novoselov KS, Geim AK, Morozov SV, Jiang D, Zhang Y, Dubonos SV, Grigorieva IV, FirsovA A (2004) Electric field effect in atomically thin carbon films. Science 306:666-669
2. Novoselov KS, Geim AK, Morozov SV, Jiang D, Katsnelson MI, Grigorieva IV (2005) Two-dimensional gas of massless Dirac fermions in graphene. Nature 438:197-200

3. Liu YP, Lew WS, Goolaup S, Shen ZX, Sun L, Zhou TJ, Wong SK (2012) Observation of the semiconductor-metal transition behavior in monolayer graphene. Carbon 50:2273-2279

4. Novoselov KS, Jiang Z, Zhang Y, Morozov SV, Stormer HL, Zeitler U (2007) Room-temperature quantum hall effect in graphene. Science 315:5817

5. Sonin EB (2009) Effect of Klein tunnelling on conductance and shot noise in ballistic graphene. Phys Rev B 79(19):195438

6. Serafini P, Milani A, Proserpio DM, Casari CS (2021) Designing All Graphdiyne Materialsas Graphene Derivatives: Topologically driven modulation of electronic properties. J Phys Chem C 125(33): 18456-18466. https://doi.org/10.1021/acs.jpcc.1c04238

7. Khamidullin T, Lounev I, Solodov A, Vakhitov I, Hashemi SA, Dimiev AM (2021) Graphene Oxide-Epoxy Composites with Induced Anisotropy of Electrical Properties. J Phys Chem C 125(48):26823-26831. https://doi.org/10.1021/acs.jpcc.1c07645

8. Pisani WA, Wedgeworth DN, Roth MR, Newman JK, Shukla MK (2021) Computational prediction of mechanical properties of PA6graphene/carbon nanotube nanocomposites. J Phys Chem C 125(28):15569-15578. https://doi.org/10.1021/acs.jpcc.1c03410

9. Jabed MA, Zhao J, Kilin D, Yu T (2021) Understanding of light absorption properties of the $\mathrm{N}$-doped graphene oxide quantum dot with TD-DFT. J Phys Chem C 125(27):14979-14990. https://doi. org/10.1021/acs.jpcc.1c03012

10. Stavrou M, Papadakis I, Bawari S, Narayanan TN, Couris S (2021) Giant broadband (450-2300 nm) optical limiting and enhancement of the nonlinear optical response of some Graphenes by defect engineering. J Phys Chem C 125(29):16075-16085. https://doi. org/10.1021/acs.jpcc.1c05495

11. Manoharan AK, Chinnathambi S, Jayavel R, Hanagata N (2017) Simplified detection of the hybridized DNA using a graphene field effect transistor. Sci Technol Adv Mater 18:43-50

12. Dong X, Shi Y, Huang W (2010) Electrical detection of DNA hybridization with Single-Base specificity using transistors based on CVD-grown graphene sheets. Adv Mater 22:1649-1653

13. Meric I, Dean CR, Young AF, Baklitskaya N, Tremblay NJ, Nuckolls C, Kim P, Shepard KL (2011) Channel length scaling in graphene field-effect transistors studied with pulsed current-voltage measurements. Nano Lett 11(3):1093-1097

14. Novoselov KS, Falko VI, Colombo L, Gellert PR, Schwab MG, Kim K (2012) A roadmap for graphene. Nature 490:192-200

15. Cranford SW, Buehler MJ (2011) Mechanical properties of graphyne. Carbon 49(13):4111-4121

16. Hess LH, Jansen M, Maybeck V, Hauf MV, Seifert M, Stutzmann M, Sharp LD, Offenhausser A, Garrido JA (2011) Graphene transistor arrays for recording action potentials from electrogenic cells. Adv Mater 23:5045-5049

17. Hess LH, Lyuleeva A, Blaschke BM, Sachsenhauser M, Seifert M, Garrido JA (2014) Graphene transistors with multifunctional polymer brushes for biosensing applications. ACS Appl Mater Interfaces 6:9705-9710

18. Wang S, Wu Z, Zheng S, Zhou F, Sun C, Cheng H, Bao X (2017) Scalable fabrication of Photochemically reduced graphene-based monolithic Micro-supercapacitors with superior energy and power densities. ACS Nano 11(4):4283-4291

19. Zheng S, Tang X, Wu Z, Tan Y, Wang S, Sun C, Cheng H, Bao X (2017) Arbitrary-shaped graphene-based planar Sandwich supercapacitors on one substrate with enhanced flexibility and integration. ACS Nano 11(2):2171-2179

20. Lang F, Gluba MA, Albrecht S, Rappich J, Korte L, Rech B, Nickel NH (2015) Perovskite solar cells with large-area CVD-graphene for tandem solar cells. J Phys Chem Lett 6(14):2745-2750 
21. Wu Y, Zhang X, Jie J, Xie C, Zhang X, Sun B, Wang Y, Gao P (2013) Graphene transparent conductive electrodes for highly efficient silicon nanostructures-based hybrid heterojunction solar cells. J Phys Chem C 117(23):11968-11976

22. Lu CH, Yang HH, Zhu CL (2009) A graphene platform for sensing biomolecules. Angew Chem 48:4785-4787

23. Huang Y, Dong X, Shi Y (2010) Nanoelectronic biosensors based on CVD grown graphene. Nanoscale 2:1485-1488

24. Beraud A, Sauvage M, Bazan CM, Tie M, Bencherif A, Bouilly D (2021) Graphene field-effect transistors as bioanalytical sensors: design, operation and performance. Analyst 146:403-428

25. Vamsi Krsihna B, Ahmadsaidulu S, Teja SST, Jayanthi D, Navaneetha A, Reddy PR, Prakash MD (2021) Design and development of graphene FET biosensor for the detection of SARSCoV-2. Silicon 12:1-9

26. Zhang X et al (2020) Electrical probing of COVID-19 spike protein receptor binding domain via a graphene field-effect transistor. ArXiv:2003.12529 [Cond-Mat, Physics: Physics]

27. Kumar Manoharan A, Jayavel R, Shanmugam M, Sengottaiyan C, Chinnathambi S, Mohankumar N (2021) A compact sensory platform based $\mathrm{pH}$ sensor using graphene field effect transistor. J Nanosci Nanotechnol 21(6):3299-3305

28. Venugopal A, Chan J, Li X, Magnuson CW, Kirk WP, Colombo L, Ruoff RS, Vogel EM (2011) Effective mobility of single-layer graphene transistors as a function of channel dimensions. J Appl Phys 109:104511
29. Lin CT, Loan PTK, Chen TY (2013) Label-free electrical detection of DNA hybridization on graphene using hall effect measurements: revisiting the sensing mechanism. Adv Funct Mater 23:2301-2307

30. Lafkioti M, Krauss B, Lohmann T, Zschieschang U, Klauk H, Klitzing KV, Smet JH (2010) Graphene on a hydrophobic substrate: doping reduction and hysteresis suppression under ambient conditions. Nano Lett 10:1149-1153

31. Uesugi E, Goto H, Eguchi R (2013) Electric double-layer capacitance between an ionic liquid and few-layer graphene. Sci Rep 3: 1595

32. Jung H, Park J, Oh I-K (2014) Fabrication of transferable $\mathrm{Al}_{2} \mathrm{O}_{3}$ Nanosheet by atomic layer deposition for graphene FET. ACS Appl Mater Interfaces 6:2764-2769

33. Ohno Y, Okamoto S, Maehashi K, Matsumoto K (2013) Direct electrical detection of DNA hybridization based on electrolytegated graphene field-effect transistor. Jpn J Appl Phys 52:110107

34. Moser J, Barreiro A, Bachtold A (2007) Current induced cleaning of graphene. Appl Phys Lett 91:163513

35. Lee MH, Kim BJ, Lee KH, Shin LS, Huh W, Cho JH, Kang MS (2015) Apparent pH sensitivity of solution gated graphene transistors. Nanoscale 7:7540-7544

Publisher's Note Springer Nature remains neutral with regard to jurisdictional claims in published maps and institutional affiliations. 